\title{
A Class of Migration Algorithms for Ground-Penetrating Radar Data
}

\author{
Richard G. Plumb ${ }^{1}$ and Carl Leuschen ${ }^{2}$ \\ ${ }^{1}$ Department of Electrical Engineering, State University of New York at Binghamton, \\ P.O. Box 6000 Binghamton, NY 13902 USA \\ Phone: 607-777-4846 Fax: 607-777-4464 email: rplumb@binghamton.edu \\ ${ }^{2}$ The University of Kansas, Radar Systems and Remote Sensing Laboratory \\ 2291 Irving Hill Road, Lawrence, KS 66045 \\ Phone: 785-864-7739, Fax: 785-864-7789, email: leuschen@rsl.ukans.edu
}

\begin{abstract}
Ground-penetrating radar (GPR) is a mature remote sensing technique used to obtain spatial location and reflectivity information on subsurface features from data collected over the surface. Various imaging or migration techniques have been used to refocus the scattered signals from the $x$ - $t$ domain or image space back to their true spatial location in the object space. The goal of this presentation is to outline a class of generalized GPR migration algorithms that are firmly based on the electromagnetic theory and radar principles. The development of these algorithms is based on a matched-filter response. By presenting migration in terms of a matched filter and electromagnetic theory, further understanding of the physical processes involved within GPR collection and imaging are gained. In particular, we present the necessary assumptions required for the development and application of a class of two- and three-dimensional vector migration algorithms for bistatic and monostatic radar configurations. Finally, a bistatic three-dimensional polarimetric simulation is presented using the finite-difference time-domain.
\end{abstract}

\section{INTRODUCTION}

Since most GPRs use broad beamwidth antennas, the energy reflected from a buried structure is recorded over a large lateral aperture. As a result, the only information that can be directly interpreted from a single time response is range. Wave migration is a common application that is used to refocus these time responses, which were recorded along an aperture on the surface, to their true spatial location. This transformation into the $x$ - $z$ domain redistributes the energy in such a manner that these scattering events are migrated to their location within the ground.

Many of the migration algorithms used for GPR originated within the geophysical community and were developed with seismic applications in mind. Even though the wave excitation, scattering, and measurement associated with these two practices are quite different, these techniques are readily used because they often accomplish the initial goal of producing an image. However, there still exists certain assumptions and approximations that allows their use to be extended to GPR. In the following section, a matched-filter migration algorithm is developed for GPR applications.
The development of this algorithm is based on a matched filter. The idea of a matched filter can be explained as the correlation of the received signal with an expected or estimated signal from a specific target. If this correlation produces a large value, then it is likely that the target is present. The implementation of the matched filter involves an estimation of this expected signal and an interpretation of the convolution. When linear scattering theory is used to determine the expected signal and additional assumptions are made, the resulting convolution takes on the form of a forward scattering problem.

\section{DEVELOPMENT}

The implementation of a matched filter includes estimating the expected response, $H(\omega)$, and convolving this response with the recorded data. In the development of the presented algorithm, this expected response is determined from the scattered field from a point target within the ground. Referring to Fig. 1, $\mathbf{r}^{\prime \prime}$ is the location of the transmit antenna, $\mathbf{r}^{\prime}$ is the location of the point scatterer, and $\mathbf{r}$ is the location of the receive antenna. Using vector notation and assuming point-like apertures, the scattered field can be written as [1]

$$
H\left(\mathbf{r}^{\prime \prime}, \mathbf{r}^{\prime}, \mathbf{r}, \omega\right)=\mathbf{u}_{r} \overline{\mathbf{G}}_{r}\left(\mathbf{r}, \mathbf{r}^{\prime}, \omega\right) \overline{\mathbf{O}}\left(\mathbf{r}^{\prime}\right) \overline{\mathbf{G}}_{i}\left(\mathbf{r}^{\prime}, \mathbf{r}^{\prime \prime}, \omega\right) \mathbf{u}_{t}
$$

where $\mathbf{u}_{t}$ and $\mathbf{u}_{r}$ are the polarizations of the transmit and receive antennas, respectively, $\mathbf{G}$ is the electric-field dyadic Green's function [2], and $\mathbf{O}$ is the object scattering matrix.

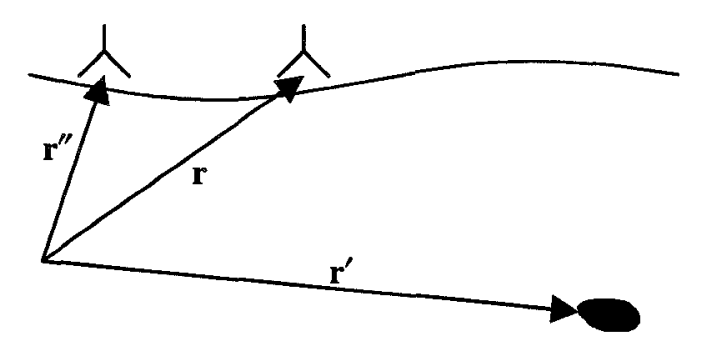

Fig. 1. Basic ground-penetrating radar configuration with transmit antenna, $\mathbf{r}^{\prime \prime}$, receive antenna, $\mathbf{r}$, and scatterer, $\mathbf{r}^{\prime}$. 
The dyadic Green's function expresses the electric field due to an impulsive current source, while the object scattering matrix relates the induced current within the object to an incident electric field. In most cases only the diagonal components of the object scattering matrix are considered, but for polarimetric applications, the off-diagonal components must also be included.

\section{Bistatic Configuration}

When the recorded signals, $R(\mathbf{r}, \omega)$, of a bistatic survey are applied to the filter shown in (1), the migrated image, $S\left(r^{\prime}\right)$, is expressed as a convolution of two fields evaluated at time zero. This is equivalent to multiplication and integration in the frequency domain,

$$
S\left(\mathbf{r}_{s}^{\prime}\right)=\int \mathbf{E}_{b p}\left(\mathbf{r}^{\prime}, \omega\right) \cdot \mathbf{E}_{i n c}\left(\mathbf{r}^{\prime}, \omega\right) d \omega
$$

where

$$
\mathbf{E}_{b p}\left(\mathbf{r}^{\prime}, \omega\right)=\sum_{m=1}^{M} \overline{\mathbf{G}}\left(\mathbf{r}^{\prime}, \mathbf{r}_{m}, \omega\right) \cdot\left[R^{*}\left(\mathbf{r}_{m}, \omega\right)\right]_{\mathbf{r}_{r}}
$$

is referred to as the back-propagated electric field and

$$
\mathbf{E}_{i n c}\left(\mathbf{r}^{\prime}, \omega\right)=\overline{\mathbf{G}}\left(\mathbf{r}^{\prime}, \mathbf{r}^{\prime \prime}, \omega\right) \cdot T(\omega) \mathbf{u}_{t}
$$

is the incident electric field. Here, $T(\omega)$ is the transmit waveform. The back-propagated field is interpreted as a means of propagating the scattered fields back to the original object locations within the ground. This field is calculated by reversing the recorded data in time and reintroducing these time-reversed signals as current sources at their respective receiver locations. Since superposition of current sources applies to electromagnetic waves, the back-propagated field usually accounts for the recorded data from a complete receiver array, shown by the summation over $m$ in (3).

\section{Monostatic Configuration}

When the recorded signals of a monostatic survey are applied to the filter shown in (1), the migrated image is expressed by the back-propagated field only.

$$
\left.\mathbf{E}_{b p}\left(\mathbf{r}^{\prime}\right)=\int \sum_{m=1}^{M} \overline{\mathbf{G}}\left(2 \mathbf{r}^{\prime}, 2 \mathbf{r}_{m}, \omega\right) \cdot\left[R^{*}\left(\mathbf{r}_{m}, \omega\right)\right]\right]_{r} d \omega
$$

This is a direct result of the "exploding reflector model" [3], which provides an alternative explanation of the scattering processes involved within a monostatic configuration. More specifically, this equivalent model is represented by removing the transmit antenna, replacing the scattering objects by simultaneously stimulated sources, and increasing all the dimensions by a factor of two. The model accounts for the two-way travel time of the original configuration by a oneway travel time across twice the distance.

\section{Polarization}

As stated earlier, the object scattering matrix can be modified to test for depolarization characteristics of the object. For an object with no depolarization characteristics, the object scattering matrix would be the identity matrix, while the off-diagonal components would account for various types of depolarization. For example, an object that depolarizes an $\mathrm{x}$-directed electric field into $\mathrm{y}$-directed induced currents would contain an object scattering matrix of the form

$$
\overline{\mathbf{O}}=\left[\begin{array}{lll}
0 & 0 & 0 \\
1 & 0 & 0 \\
0 & 0 & 0
\end{array}\right]
$$

The implication of the object scattering matrix on the migration algorithm is seen in the convolution operation. The matrix defines which component of the incident and backpropagated vector fields are to be convolved. For the matrix shown in (6), the x-component of the incident field would be convolved with the y-component of the back-propagated field.

\section{VARIATIONS OF THE MIGRATION ALGORITHM}

Different variations of this migration algorithm are a result of the specific method used to propagate the incident and back-propagated fields. In general, any forward scattering technique can be used to evaluate these fields, but some techniques will offer specific advantages in certain applications. For example, if a large area is to be imaged, a simple scalar forward scattering technique may be necessary due to time and memory constraints, while if a small area in the near field of the transmit or receive antenna is to be investigated, a more complicated vector solution of the wave equation may produce more accurate results.

\section{SIMULATION}

A three-dimensional simulation was conducted using the finite-difference time-domain (FDTD) method as the means of wave propagation. The FDTD method provides a solution of the vector wave equation allowing the inclusion of polarimetric measurement. Additionally, the FDTD method allows any a priori knowledge such as elevation changes or inhomogeneities to be included into the simulation.

As shown in Fig. 2, the FDTD space consists of a homogeneous ground, with a relative permittivity of four, and two $0.75-\mathrm{m}$ perpendicular conducting rods, which are modeled by forcing the tangential electric field to zero. The first rod is at a depth of $0.3 \mathrm{~m}$ and oriented in the y-direction, while the second rod is at a depth of $0.45 \mathrm{~m}$ and oriented in the $x$-direction. Four sets of measurements were conducted including $x-x, x-y, y-x$, and $y-y$ polarizations of the receive and transmit antennas, respectively. 


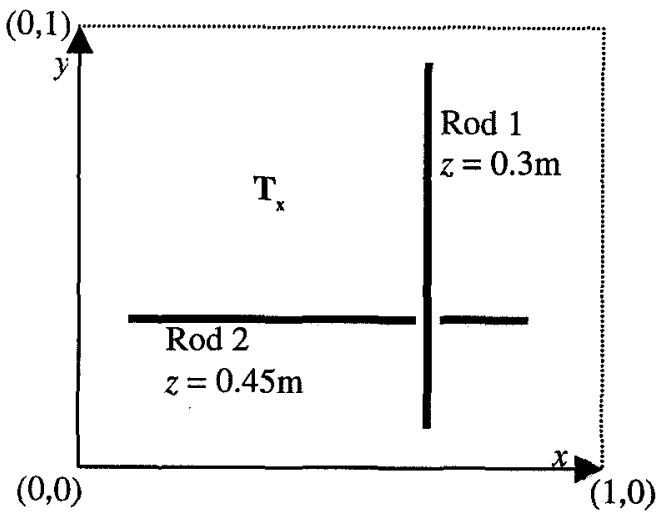

Fig. 2. Top view of the FDTD configuration.

The transmit antenna was located at $\mathrm{x}$ equal to $0.55 \mathrm{~m}$ and $\mathrm{y}$ equal to $0.55 \mathrm{~m}$, while a two-dimensional receiving array was spaced every $3 \mathrm{~cm}$ on the surface. For every receive-transmit polarization, an appropriate model was chosen for the scattering matrix as shown in (7)-(10).

$\mathrm{X}-\mathrm{X}$ receive-transmit polarization:

$$
\overline{\mathbf{O}}=\left[\begin{array}{lll}
1 & 0 & 0 \\
0 & 0 & 0 \\
0 & 0 & 0
\end{array}\right]=o_{x x},
$$

$\mathrm{x}-\mathrm{y}$ receive-transmit polarization:

$$
\overline{\mathbf{O}}=o_{x y}
$$

$\mathrm{y}-\mathrm{x}$ receive-transmit polarization:

$$
\overline{\mathbf{O}}=o_{y x}
$$

y-y receive-transmit polarization:

$$
\overline{\mathbf{O}}=o_{y y},
$$

\section{RESULTS AND CONCLUSIONS}

The resulting images are shown in Fig. 3 for the y-directed rod and Fig. 4 for the $\mathrm{x}$-directed rod. In each figure there are four images for each receive-transmit polarization combination. Several things can be seen in these images. First, as expected, the $\mathrm{x}$-directed rod has a strong tendency to induce currents in the $\mathrm{x}$-direction, while the $\mathrm{y}$-directed rod has a strong tendency to induce currents in the $y$-direction. Another interesting result is seen in the $y-y$ image for the second rod (lower-right image in Fig. 4). Although the directed rod exists at this depth, use of the $y-y$ polarization brought out a sidelobe of the higher $y$-directed rod. We have discussed a class of GPR migration algorithms, developed on a matched-filter response basis, in order to understand GPR physical processes better. We have shown necessary assumptions for various radar configurations and presented a bistatic, 3-D polarimetric simulation using FDTD.
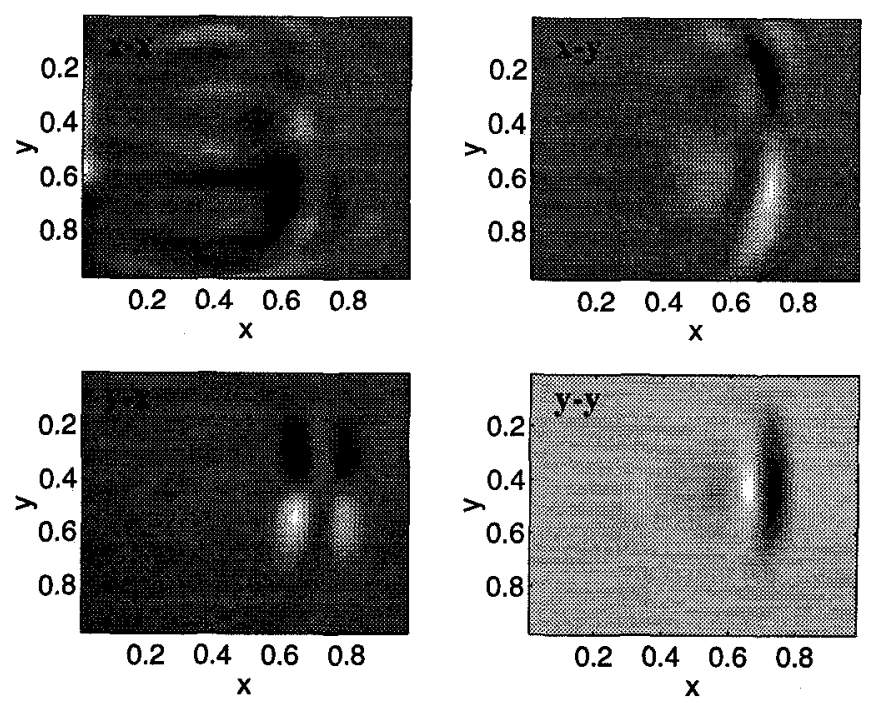

Fig. 3. Images for the $y$-directed rod at a depth of 0.3 meters. $x-x$ (top left), $x-y$ (top right), $y-x$ (bottom left), and $y-y$ (bottom right).
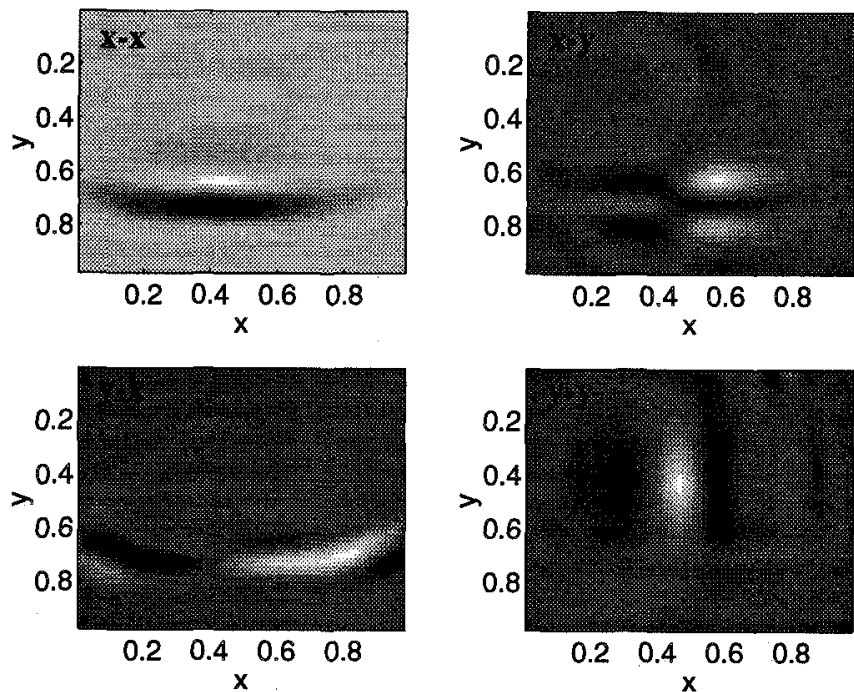

Fig. 4. Images for the $x$-directed rod at a depth of 0.45 meters. $x-x$ (top left), $x-y$ (top right), $y-x$ (bottom left), and $y-y$ (bottom right).

\section{REFERENCES}

[1] C Leuschen. and R. Plumb, "A matched-filter-based reverse-time-migrating algorithm for ground-penetrating radar data," unpublished.

[2] W. C. Chew, Waves and Fields in Inhomogeneous Media, Van Nostrand Reinhold: New York, 1990.

[3] D. Loewenthal, L. Lu, R. Roberson, and J. Sherwood, "The wave equation applied to migration," Geophysical Prospecting, vol. 24, no. 2, pp. 380-399, June 1976. 